\title{
Radar and Communication Coexistence Enabled by Interference Exploitation
}

\author{
Fan Liu* ${ }^{* \dagger}$, Christos Masouros ${ }^{\dagger}$, Ang $\mathrm{Li}^{\dagger}$ and Tharmalingam Ratnarajah ${ }^{\ddagger}$ \\ ${ }^{*}$ School of Information and Electronics, Beijing Institute of Technology, Beijing, China \\ ${ }^{\dagger}$ Department of Electronic and Electrical Engineering, University College London, London, UK \\ ${ }_{\ddagger}^{\ddagger}$ Institute for Digital Communications, School of Engineering, The University of Edinburgh, Edinburgh, UK \\ Email: liufan92@bit.edu.cn, chris.masouros@ieee.org, ang.li.14@ucl.ac.uk, t.ratnarajah@ed.ac.uk
}

\begin{abstract}
In this paper, we propose a novel approach for the spectrum sharing between Multi-Input-Multi-Output (MIMO) radar and downlink multi-user Multi-Input-Single-Output (MUMISO) communication system. To obtain a power-efficient beamforming at the base station (BS), we utilize the constructive multi-user interference (MUI) as a source of green signal power. The proposed beamforming design mainly focuses on two optimization problems, i.e., transmit power minimization for BS and interference minimization for radar, subject to given performance requirements of the two systems. We further consider the impact of the proposed methods on radar, where the detection probability for MIMO radar in the presence of the interference from BS is analytically derived, and important trade-offs are revealed. Numerical results show that the proposed approach outperforms the conventional beamforming designs by achieving a significant performance gain under the discussed coexistence scenario.
\end{abstract}

Index Terms-MU-MISO downlink, radar-communication coexistence, spectrum sharing, constructive interference.

\section{INTRODUCTION}

The increasing need for spectrum for wireless communications, has stimulated significant governmental and spectrum regulators' activities to the direction of providing commercial communications with access to the radar spectrum [1]-[3]. The fact that most of the latter occupies the frequency bands below X-band, makes it particularly desirable. Recently, the coexistence schemes for the two systems have drawn much attention from the researchers. In [4], Opportunistic Spectrum Sharing (OSS) between radar and cellular has been considered, where the communication system transmits signals when the space and frequency spectra are not occupied by radar. More fundamentally, efforts have been taken to unify radar and communication under the framework of information theory [5], where an estimation rate for radar has been defined using rate distortion theory. Nevertheless, the above works focus on single-antenna systems, rather than MIMO systems. So far, several approaches have been proposed to enable the coexistence between MIMO radar and communications. The pioneered work [6] proposes a null space projection (NSP) beamforming approach, which has been further studied by [7]-[9]. Besides, the optimization-based beamforming for the coexistence has been investigated in [10], [11]. While research progress has been made on the coexistence between radar and Point-to-Point (P2P) communication coexistence, little attention has been paid to the case of radar and multiuser communications. In view of this, previous work [12] considers the robust MIMO beamforming for the coexistence of radar and downlink MU communication using Semidefinite Relaxation (SDR) optimizations [13], [14]. However, the SDR-based beamforming has been shown to be suboptimal by recent works [15], [16], where an optimization approach based on interference exploitation has been given.

As per the concept of the constructive interference (CI), this work develops an optimization-based beamforming method for the spectrum sharing between MIMO radar and MUMISO communication. The interference exploitation approach is first introduced in [17]-[19] to design closed-form beamformers. Recent progress [15], [16] shows that by rotating the destructive interference into constructive region using optimization techniques, the receive Signal-to-Interferenceplus-Noise-Ratio (SINR) at the users is increased. By contrast, it can be further shown that for a given SINR constraint using constructive interference, the feasible domain of the optimization problem is relaxed compared to the conventional schemes.

In this paper, we mainly focus on two optimization-based transmit beamforming designs at the BS: 1) Transmit power minimization at the BS while guaranteeing the receive SINR at the users and the interference level from BS to radar; 2) Interference minimization for radar subject to the SINR constraint per user and transmit power budget at the BS. By analytically deriving the detection probability in the presence of the interference from the BS, we further investigate the effect of interference minimization beamforming on the performance of radar, through which important trade-offs between radar and communication are given. Numerical simulations show significant performance gain of the proposed scheme over the conventional SDR-based approach.

\section{SySTEM MODEL}

We consider a coexistence scenario where a $K$-user MUMISO downlink system operates at the same frequency band with a MIMO radar. As shown in Fig. 1, the $N$-antenna BS is transmitting signals to $K$ single-antenna users while the MIMO radar with $M_{t}$ transmit antennas and $M_{r}$ receive antennas is detecting a point-like target in the far-field. The 


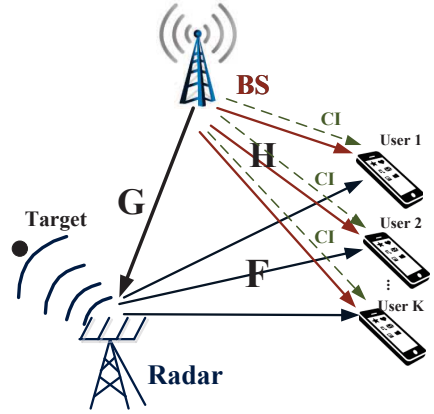

Fig. 1. Spectrum sharing scenario.

received signal at the $i$-th user is given as

$$
y_{i}^{C}[l]=\mathbf{h}_{i}^{T} \sum_{k=1}^{K} \mathbf{t}_{k} d_{k}[l]+\sqrt{P_{R}} \mathbf{f}_{i}^{T} \mathbf{s}_{l}+n_{i}[l], i=1,2, \ldots, K,
$$

where $\mathbf{h}_{i} \in \mathbb{C}^{N \times 1}$ denotes the communication channel vector, $\mathbf{f}_{i} \in \mathbb{C}^{M_{t} \times 1}$ denotes the interference channel vector from radar to the user, $\mathbf{t}_{i} \in \mathbb{C}^{N \times 1}$ denotes the precoding vector, $d_{i}[l]$ and $n_{i}[l] \sim \mathcal{C N}\left(0, \sigma_{C}^{2}\right)$ stands for the communication symbol and the received noise for the $i$-th user. $l=1,2, \ldots, L$ is the symbol index, $L$ is the length of the communication frame, and $P_{R}$ is the power of radar signal. Without loss of generality, we assume that the communication symbol is drawn from a normalized PSK constellation, where the PSK symbol can be denoted as $d_{k}[l]=e^{j \phi_{k}[l]}$, while we note that the proposed concept of interference exploitation has been shown to offer benefits for other modulation formats [20], [21]. It is assumed that $\mathbf{H}=\left[\mathbf{h}_{1}, \mathbf{h}_{2}, \ldots, \mathbf{h}_{K}\right]$ and $\mathbf{F}=\left[\mathbf{f}_{1}, \mathbf{f}_{2}, \ldots, \mathbf{f}_{K}\right]$ are flat Rayleigh fading and statistically independent with each other, and can be estimated by the BS through the pilot symbols.

The second term at the right hand of (1) is the interference from radar to the user, where $\mathbf{S}=\left[\mathbf{s}_{1}, \mathbf{s}_{2}, \ldots, \mathbf{s}_{L_{R}}\right] \in \mathbb{C}^{M_{t} \times L_{R}}$ is the radar transmit waveforms with $\mathbf{s}_{l}$ being the $l$-th snapshot across the radar antenna array. According to the standard assumption in MIMO radar literature [22], [23], $\mathbf{S}$ is set to be orthogonal, i.e., $\frac{1}{L_{R}} \sum_{l=1}^{L_{R}} \mathbf{s}_{l} \mathbf{s}_{l}^{H}=\mathbf{I}$. For the ease of our derivation, we also assume $L_{R}=L$, while it should be highlighted that in order to preserve the orthogonality of $\mathbf{S}$, radar may utilize codeword that is longer than a typical communication frame.

Based on the above, the receive SINR for the $i$-th downlink user is given by

$$
\gamma_{i}=\frac{\left|\mathbf{h}_{i}^{T} \mathbf{t}_{i}\right|^{2}}{\sum_{k=1, k \neq i}^{K}\left|\mathbf{h}_{i}^{T} \mathbf{t}_{k}\right|^{2}+P_{R}\left\|\mathbf{f}_{i}\right\|^{2}+\sigma_{C}^{2}}, \forall i
$$

And the average transmit power of the BS is

$$
P_{C}=\sum_{k=1}^{K}\left\|\mathbf{t}_{k}\right\|^{2}
$$

In the presence of a point-like target located at direction $\theta$, the echo wave received by radar at the $l$-th snapshot is

$$
\mathbf{y}_{l}^{R}=\alpha \sqrt{P_{R}} \mathbf{A}(\theta) \mathbf{s}_{l}+\mathbf{G}^{T} \sum_{k=1}^{K} \mathbf{t}_{k} d_{k}[l]+\mathbf{z}_{l},
$$

where $\mathbf{G}=\left[\mathbf{g}_{1}, \mathbf{g}_{2}, \ldots, \mathbf{g}_{M_{r}}\right] \in \mathbb{C}^{N \times M_{r}}$ is the interference channel matrix between BS and radar RX, and is also assumed to be flat Rayleigh fading and statistically independent with other two channels, and is estimated at the BS, $\alpha \in \mathbb{C}$ is the complex path loss of the path between radar and target, $\mathbf{z}_{l}=\left[z_{1}[l], z_{2}[l], \ldots, z_{M_{r}}[l]\right]^{T} \in \mathbb{C}^{M_{r} \times 1}$ is the received noise at the $l$-th time slot with $z_{m}[l] \sim \mathcal{C N}\left(0, \sigma_{R}^{2}\right), \forall m$, $\mathbf{A}(\theta)=\mathbf{a}_{R}(\theta) \mathbf{a}_{T}^{T}(\theta)$, in which $\mathbf{a}_{T}(\theta) \in \mathbb{C}^{M_{t} \times 1}$ and $\mathbf{a}_{R}(\theta) \in \mathbb{C}^{M_{r} \times 1}$ are transmit and receive steering vectors of the radar antenna array. The model in (4) is assumed to be obtained in a single range-Doppler bin of the radar detector and thus omits the range and Doppler parameters. In this paper, we apply the basic assumptions in [23] on the radar model, which is

$$
\begin{aligned}
& M_{r}=M_{t}=M, \quad \mathbf{a}_{R}(\theta)=\mathbf{a}_{T}(\theta)=\mathbf{a}(\theta), \\
& \mathbf{A}_{i m}(\theta)=\mathbf{a}_{i}(\theta) \mathbf{a}_{m}(\theta)=e^{-j \omega \tau_{i m}(\theta)} \\
& =e^{\left(-j \frac{2 \pi}{\lambda}[\sin (\theta) ; \cos (\theta)]^{T}\left(\mathbf{x}_{i}+\mathbf{x}_{m}\right)\right)},
\end{aligned}
$$

where $\omega$ and $\lambda$ denote the frequency and the wavelength of the carrier, $\mathbf{A}_{i m}(\theta)$ is the $i$-th element at the $m$-th column of the matrix $\mathbf{A}$, which is the total phase delay of the signal that transmitted by the $i$-th element and received by the $m$-th element of the antenna array, and $\mathbf{x}_{i}=\left[x_{i}^{1} ; x_{i}^{2}\right]$ is the location of the $i$-th element of the antenna array. In the above radar signal model, the communication interference is assumed to be the only interference received by radar. Following the closely related literature, the interference caused by clutter and false targets is not considered [7]. For convenience, we omit the time index $l$ in the rest of the paper unless otherwise specified.

\section{PROBLEM Formulation}

\section{A. SDR-based Beamforming}

Let us first define the Interference-to-Noise-Ratio (INR) at the $m$-th receive antenna of radar as

$$
r_{m}=\left|\mathbf{g}_{m}^{T} \sum_{k=1}^{K} \mathbf{t}_{k} d_{k}\right|^{2} / \sigma_{R}^{2}
$$

The conventional power minimization problem of the BS subject to INR and SINR thresholds is then formulated as

$$
\begin{array}{ll}
\mathcal{P}_{0}: \min _{\mathbf{t}_{k}} P_{C} \\
\text { s.t. } & \gamma_{i} \geq \Gamma_{i}, \forall i, \\
& r_{m} \leq R_{m}, \forall m,
\end{array}
$$

where $\Gamma_{i}$ is the required SINR of the $i$-th communication user, $R_{m}$ is the maximum tolerable INR level of the $m$-th receive element of radar. Similarly, we can formulate the optimization problem that minimizes the interference to radar 


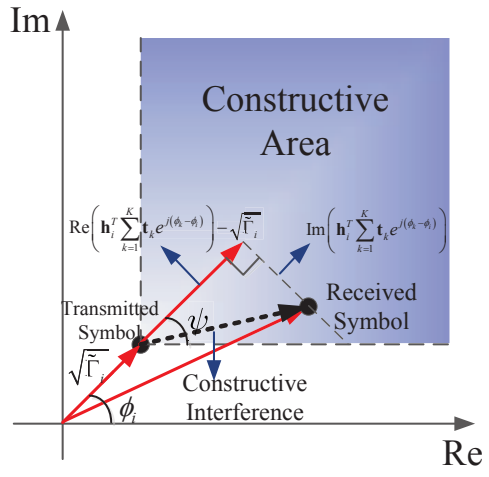

Fig. 2. The principle of constructive interference.

while guaranteeing the BS power budget and the required SINR level at each user, which is given as

$$
\begin{array}{ll}
\mathcal{P}_{1}: \min _{\mathbf{t}_{k}} \sum_{m=1}^{M}\left|\mathbf{g}_{m}^{T} \sum_{k=1}^{K} \mathbf{t}_{k} d_{k}\right|^{2} \\
\text { s.t. } & P_{C} \leq P, \\
& \gamma_{i} \geq \Gamma_{i}, \forall i,
\end{array}
$$

where $P$ is the budget of the BS transmit power. Problem $\mathcal{P}_{0}$ and $\mathcal{P}_{1}$ can be transformed into Semidefinite Program (SDP) [24] with Semidefinite Relaxation techniques, and thus can be solved by numerical tools. We refer readers to [12]-[14] for more details on this topic. As shown in Fig. 1 by green dashed arrows, it is worth noting the above problems ignore the fact that for each user, interference from other users can contribute to the received signal power constructively. In this paper, we aim to show that the solution of these problems is suboptimal from an instantaneous point of view and design a symbol-based beamforming method in accordance to the concept of constructive interference.

\section{B. Beamforming with Constructive Interference}

The instantaneous interference can be divided into two categories, constructive interference and destructive interference. Generally, the constructive interference is defined as the interference that moves the received symbol away from the decision thresholds. The purpose of the CI-based beamforming is to rotate the known interference from other users such that the resultant received symbol falls into the constructive region. This is shown in Fig. 2, where QPSK constellation is employed as an example and we denote the constructive area by the gray shade. It has been proven in [16] that the CI-based optimization will become more relaxed than conventional optimizations due to the expansion of the feasible region. Hence, the performance of the beamformer is improved. Here we consider the instantaneous transmit power, which is given as

$$
P_{T}[l]=\left\|\sum_{k=1}^{K} \mathbf{t}_{k} e^{j\left(\phi_{k}[l]-\phi_{1}[l]\right)}\right\|^{2},
$$

where $d_{1}[l]=e^{j \phi_{1}[l]}$ is used as the phase reference. Based on [16], we rewrite the SINR constraints of $\mathcal{P}_{0}$ and $\mathcal{P}_{1}$ in a CI sense, and reformulate the power minimization problem $\mathcal{P}_{0}$ as (11) on the top of the next page as $\mathcal{P}_{2}$, where $\psi=\frac{\pi}{M_{p}}$, and $M_{p}$ is the PSK modulation order. Readers are referred to [16] for a detailed derivation of the CI constraints and classification. Note that $\mathcal{P}_{2}$ is convex in contrast to the nonconvex $\mathcal{P}_{0}$ and $\mathcal{P}_{1}$. To be more specific, problem $\mathcal{P}_{2}$ is a second-order cone program (SOCP) and can be solved optimally by numerical tools. Following the virtual multicast model in [16], the power minimization problem $\mathcal{P}_{2}$ can be equivalently written as

$$
\begin{aligned}
& \mathcal{P}_{3}: \min _{\mathbf{w}}\|\mathbf{w}\|^{2} \\
& \text { s.t. }\left|\operatorname{Im}\left(\tilde{\mathbf{h}}_{i}^{T} \mathbf{w}\right)\right| \leq\left(\operatorname{Re}\left(\tilde{\mathbf{h}}_{i}^{T} \mathbf{w}\right)-\sqrt{\tilde{\Gamma}_{i}}\right) \tan \psi, \forall i, \\
&\left|\tilde{\mathbf{g}}_{m}^{T} \mathbf{w}\right| \leq \sqrt{R_{m} \sigma_{R}^{2}}, \forall m,
\end{aligned}
$$

where $\mathbf{w} \triangleq \sum_{k=1}^{K} \mathbf{t}_{k} e^{j\left(\phi_{k}-\phi_{1}\right)}, \tilde{\mathbf{h}}_{i} \triangleq \mathbf{h}_{i} e^{j\left(\phi_{1}-\phi_{i}\right)}, \tilde{\mathbf{g}}_{m} \triangleq$ $\mathbf{g}_{m} e^{j \phi_{1}}, \tilde{\Gamma}_{i}=\Gamma_{i}\left(\sigma_{C}^{2}+P_{R}\left\|\mathbf{f}_{i}\right\|^{2}\right)$. Similarly, the CI-based interference minimization problem is given by

$$
\begin{aligned}
& \mathcal{P}_{4}: \min _{\mathbf{w}} \sum_{m=1}^{M}\left|\tilde{\mathbf{g}}_{m}^{T} \mathbf{w}\right|^{2} \\
& \text { s.t. }\left|\operatorname{Im}\left(\tilde{\mathbf{h}}_{i}^{T} \mathbf{w}\right)\right| \leq\left(\operatorname{Re}\left(\tilde{\mathbf{h}}_{i}^{T} \mathbf{w}\right)-\sqrt{\tilde{\Gamma}_{i}}\right) \tan \psi, \forall i, \\
& \quad\|\mathbf{w}\| \leq \sqrt{P} .
\end{aligned}
$$

After obtaining the optimal solution $\mathbf{w}$, the beamforming vectors can be obtained as

$$
\mathbf{t}_{k}=\frac{\mathbf{w} e^{j\left(\phi_{1}-\phi_{k}\right)}}{K}, \forall k
$$

Note that both $\mathcal{P}_{3}$ and $\mathcal{P}_{4}$ are convex and can be easily solved by numerical tools.

\section{IMPACT ON RADAR PERFORMANCE}

\section{A. SDR-based beamforming}

The interference from BS to radar will have an impact on radar's performance, which will lower the detection probability. Note that the target detection process can be described as a binary hypothesis testing problem, which is given by

$$
\mathbf{y}_{l}^{R}=\left\{\begin{array}{c}
\mathcal{H}_{1}: \alpha \sqrt{P_{R}} \mathbf{A}(\theta) \mathbf{s}_{l}+\mathbf{G}^{T} \sum_{k=1}^{K} \mathbf{t}_{k} d_{k}[l]+\mathbf{z}_{l}, \\
l=1,2, \ldots, L, \\
\mathcal{H}_{0}: \mathbf{G}^{T} \sum_{k=1}^{K} \mathbf{t}_{k} d_{k}[l]+\mathbf{z}_{l}, \quad l=1,2, \ldots, L .
\end{array}\right.
$$

We assume that the covariance matrix of interference-plusnoise has been accurately estimated by the radar. Due to the unknown parameters $\alpha$ and $\theta$, we use the Generalized Likelihood Ratio Test (GLRT) to solve the above problem. 


$$
\begin{aligned}
& \mathcal{P}_{2}: \min _{\mathbf{t}_{k}}\left\|\sum_{k=1}^{K} \mathbf{t}_{k} e^{j\left(\phi_{k}-\phi_{1}\right)}\right\|^{2} \\
& \text { s.t. }\left|\operatorname{Im}\left(\mathbf{h}_{i}^{T} \sum_{k=1}^{K} \mathbf{t}_{k} e^{j\left(\phi_{k}-\phi_{i}\right)}\right)\right| \leq\left(\operatorname{Re}\left(\mathbf{h}_{i}^{T} \sum_{k=1}^{K} \mathbf{t}_{k} e^{j\left(\phi_{k}-\phi_{i}\right)}\right)-\sqrt{\Gamma_{i}\left(\sigma_{C}^{2}+P_{R}\left\|\mathbf{f}_{i}\right\|^{2}\right)}\right) \tan \psi, \forall i, \\
& \left|\mathbf{g}_{m}^{T} \sum_{k=1}^{K} \mathbf{t}_{k} e^{j \phi_{k}}\right|^{2} \leq R_{m} \sigma_{R}^{2}, \forall m \text {. }
\end{aligned}
$$

Consider the sufficient statistic of the received signal, which is obtained by matched filtering [23], and is given by

$$
\begin{aligned}
\tilde{\mathbf{Y}} & =\frac{1}{\sqrt{L}} \sum_{l=1}^{L} \mathbf{y}_{l}^{R} \mathbf{s}_{l}^{H} \\
& =\alpha \sqrt{L P_{R}} \mathbf{A}(\theta)+\frac{1}{\sqrt{L}} \sum_{l=1}^{L}\left(\mathbf{G}^{T} \sum_{k=1}^{K} \mathbf{t}_{k} d_{k}[l]+\mathbf{z}_{l}\right) \mathbf{s}_{l}^{H}
\end{aligned}
$$

Let $\tilde{\mathbf{y}}$ be the vectorization of $\tilde{\mathbf{Y}}$, we have

$$
\begin{aligned}
\tilde{\mathbf{y}}= & \operatorname{vec}(\tilde{\mathbf{Y}}) \\
= & \alpha \sqrt{L P_{R}} \operatorname{vec}(\mathbf{A}(\theta)) \\
& +\operatorname{vec}\left(\frac{1}{\sqrt{L}} \sum_{l=1}^{L}\left(\mathbf{G}^{T} \sum_{k=1}^{K} \mathbf{t}_{k} d_{k}[l]+\mathbf{z}_{l}\right) \mathbf{s}_{l}^{H}\right) \\
\triangleq & \alpha \sqrt{L P_{R}} \mathbf{d}(\theta)+\boldsymbol{\varepsilon}
\end{aligned}
$$

where $\varepsilon \in \mathbb{C}^{M^{2} \times 1}$ is zero-mean, complex Gaussian distributed, and has the block covariance matrix as

$$
\mathbf{C}=\left[\begin{array}{ccc}
\mathbf{J}+\sigma_{R}^{2} \mathbf{I}_{M} & & \mathbf{0} \\
\mathbf{0} & & \mathbf{J}+\sigma_{R}^{2} \mathbf{I}_{M}
\end{array}\right],
$$

where $\mathbf{C} \in \mathbb{C}^{M^{2} \times M^{2}}$, and $\mathbf{J}=\mathbf{G}^{T} \sum_{k=1}^{K} \mathbf{t}_{k} \mathbf{t}_{k}^{H} \mathbf{G}^{*}$.

In [23], the GLRT detection is derived in the presence of white noise only. As shown above, $\varepsilon$ is also Gaussian distributed and has a non-white covariance matrix. Hence we apply a whitening filter for the case. It is easy to verify that $\mathbf{C}$ and $\mathbf{C}^{-1}$ are both positive-definite Hermitian matrices. We then consider the Chelosky decomposition of $\mathbf{C}^{-1}$, i.e., $\mathbf{C}^{-1}=\mathbf{U U}^{H}$, where $\mathbf{U}$ is a lower triangle matrix. By using $\mathbf{U}^{H}$ as a whitening filter, (15) can be reformulated as

$$
\tilde{\mathbf{y}}_{w}=\left\{\begin{array}{l}
\mathcal{H}_{1}: \alpha \sqrt{L P_{R}} \mathbf{U}^{H} \mathbf{d}(\theta)+\mathbf{U}^{H} \boldsymbol{\varepsilon}, \\
\mathcal{H}_{0}: \mathbf{U}^{H} \boldsymbol{\varepsilon},
\end{array}\right.
$$

where $\mathbf{U}^{H} \boldsymbol{\varepsilon} \sim \mathcal{C N}\left(0, \mathbf{I}_{M^{2}}\right)$. As per the standard GLRT decision rule, if

$$
L_{\tilde{\mathbf{y}}_{w}}(\hat{\alpha}, \hat{\theta})=\frac{p\left(\tilde{\mathbf{y}}_{w} ; \hat{\alpha}, \hat{\theta}, \mathcal{H}_{1}\right)}{p\left(\tilde{\mathbf{y}}_{w} ; \mathcal{H}_{0}\right)}>\eta,
$$

then $\mathcal{H}_{1}$ is chosen, where $p\left(\tilde{\mathbf{y}}_{w} ; \hat{\alpha}, \hat{\theta}, \mathcal{H}_{1}\right)$ and $p\left(\tilde{\mathbf{y}}_{w} ; \mathcal{H}_{0}\right)$ are the Probability Density Function (PDF) under $\mathcal{H}_{1}$ and
$\mathcal{H}_{0}$ respectively, $\hat{\alpha}$ and $\hat{\theta}$ are the maximum likelihood estimations (MLE) of $\alpha$ and $\theta$ under $\mathcal{H}_{1}$, and are given by $[\hat{\alpha}, \hat{\theta}]=\max _{\alpha, \theta} p\left(\tilde{\mathbf{y}}_{w} \mid \alpha, \theta, \mathcal{H}_{1}\right), \eta$ is the decision threshold. (19) is equivalent with the model in [23], thus the GLRT test statistic is given by

$$
\begin{aligned}
L_{\widetilde{\mathbf{y}}}(\hat{\theta}) & =\frac{\left|\mathbf{d}^{H}(\hat{\theta}) \mathbf{U} \mathbf{U}^{H} \tilde{\mathbf{y}}\right|^{2}}{\left\|\mathbf{U}^{H} \mathbf{d}(\hat{\theta})\right\|^{2}}=\frac{\left|\mathbf{d}^{H}(\hat{\theta}) \mathbf{C}^{-1} \tilde{\mathbf{y}}\right|^{2}}{\mathbf{d}^{H}(\hat{\theta}) \mathbf{C}^{-1} \mathbf{d}(\hat{\theta})} \\
& =\frac{\left|\operatorname{tr}\left(\tilde{\mathbf{Y}} \mathbf{A}^{H}(\hat{\theta}) \tilde{\mathbf{J}}-1\right)\right|^{2}}{\operatorname{tr}\left(\mathbf{A}(\hat{\theta}) \mathbf{A}^{H}(\hat{\theta}) \tilde{\mathbf{J}}-1\right)} \underset{\mathcal{H}_{0}}{\stackrel{\mathcal{H}_{1}}{\gtrless} \eta,}
\end{aligned}
$$

where $\tilde{\mathbf{J}}=\mathbf{J}+\sigma_{R}^{2} \mathbf{I}_{M}$. According to [23], the asymptotic distribution of (21) is given by

$$
\ln L_{\tilde{\mathbf{y}}}(\hat{\theta}) \sim\left\{\begin{array}{l}
\mathcal{H}_{1}: \mathcal{X}_{2}^{2}(\rho), \\
\mathcal{H}_{0}: \mathcal{X}_{2}^{2},
\end{array}\right.
$$

where $\mathcal{X}_{2}^{2}$ and $\mathcal{X}_{2}^{2}(\rho)$ are central and non-central chi-squared distributions with two Degrees of Freedom (DoFs), and $\rho$ is the non-centrality parameter, which is given by

$$
\begin{aligned}
\rho & =\frac{\left\|\alpha \sqrt{L P_{R}} \mathbf{U}^{H} \mathbf{d}(\theta)\right\|^{2}}{\sigma_{w}^{2}}=|\alpha|^{2} L P_{R} \mathbf{d}^{H}(\theta) \mathbf{U} \mathbf{U}^{H} \mathbf{d}(\theta) \\
& =|\alpha|^{2} L P_{R} \operatorname{vec}\left(\mathbf{A}^{H}(\theta)\right) \mathbf{C}^{-1} \operatorname{vec}(\mathbf{A}(\theta)) \\
& =\operatorname{SNR}_{R} \sigma_{R}^{2} \operatorname{tr}\left(\mathbf{A}(\theta) \mathbf{A}^{H}(\theta)\left(\mathbf{J}+\sigma_{R}^{2} \mathbf{I}_{M}\right)^{-1}\right),
\end{aligned}
$$

where $\sigma_{w}^{2}=1$ due to the normalized interference-plus-noise, and we define radar $\mathrm{SNR}$ as $\mathrm{SNR}_{R}=\frac{|\alpha|^{2} L P_{R}}{\sigma_{R}^{2}}$ [23]. Under Neyman-Pearson criterion [25], the detection probability is thus given as

$$
P_{D}=1-\mathfrak{F}_{\mathcal{X}_{2}^{2}(\rho)}(\eta)=1-\mathfrak{F}_{\mathcal{X}_{2}^{2}(\rho)}\left(\mathfrak{F}_{\mathcal{X}_{2}^{2}}^{-1}\left(1-P_{F A}\right)\right),
$$

where $P_{F A}$ is a given constant false alarm probability, $\mathfrak{F}_{\mathcal{X}_{2}^{2}(\rho)}$ is the non-central chi-squared CDF with 2 DoFs.

\section{B. Constructive Interference based Beamforming}

The proposed CI-based beamforming should be computed symbol by symbol, which means that the precoding vectors are functions of the time index, thus the corresponding hypothesis testing problem (15) is modified as

$$
\mathbf{y}_{l}^{R}=\left\{\begin{array}{c}
\mathcal{H}_{1}: \alpha \sqrt{P_{R}} \mathbf{A}(\theta) \mathbf{s}_{l}+\mathbf{G}^{T} \tilde{\mathbf{w}}[l]+\mathbf{z}_{l}, \\
\quad l=1,2, \ldots, L, \\
\mathcal{H}_{0}: \mathbf{G}^{T} \tilde{\mathbf{w}}[l]+\mathbf{z}_{l}, \quad l=1,2, \ldots, L,
\end{array}\right.
$$


where $\tilde{\mathbf{w}}[l]=\mathbf{w}[l] e^{j \phi_{1}[l]}$. While the exact analytic form of the distribution for $\mathbf{w}[l]$ is hard to derive, here we employ the Gaussian detector for SDR beamformer in (21). We note that for CI precoding, $\mathbf{w}[l]$ is not in general Gaussian, but our results show that this is indeed an affordable approximation, and, even with a Gaussian detector, CI-based beamformer achieves better performance at radar. Following the same procedure of the previous subsection, we have

$$
\mathbf{J}=\frac{1}{L} \sum_{l=1}^{L} \mathbf{G}^{T} \tilde{\mathbf{w}}[l] \tilde{\mathbf{w}}^{H}[l] \mathbf{G}^{*}=\frac{1}{L} \sum_{l=1}^{L} \mathbf{G}^{T} \mathbf{w}[l] \mathbf{w}^{H}[l] \mathbf{G}^{*}
$$

By substituting (26) into (23) and (24) we obtain the detection probability of CI-based beamforming method.

\section{Numerical RESUlts}

This section presents numerical results based on Monte Carlo simulations. Without loss of generality, all the channel matrices follow the standard complex Gaussian distribution, and are independent and identically distributed (i.i.d). For simplicity, we set $R_{m}=R, \Gamma_{i}=\Gamma, \forall i, \forall m$. While it is plausible that the benefits of the proposed scheme extend to various scenarios, here we assume $\alpha=P_{R}=1, N=8$, $K=M=4$, and explore the results for QPSK modulation. The power of all the noise vectors are set to be $1 \mathrm{~mW}$, i.e., $\sigma_{R}^{2}=\sigma_{C}^{2}=0 \mathrm{dBm}$. For radar configuration, an Uniform Linear Array (ULA) is equipped, and 40-digit length $\mathrm{m}$ sequences are used as radar waveform, i.e., $L=40$. In addition, the target is set to be located at the direction of $\theta=\pi / 5$. We denote the conventional SDR beamformer as 'SDR' in the figures, and the proposed beamformer based on constructive interference as ' $\mathrm{CI}$ '.

First, we compare the minimized power for the two beamforming methods in Fig. 3, under a given INR level of $6 \mathrm{~dB}$ with the increasing $\Gamma$. Unsurprisingly, the power needed for transmission increases with the growth of downlink SINR for both methods. However, thanks to the exploitation of the constructive interference, it can be easily seen that the proposed method requires a lower transmit power for given INR and SINR requirements than the conventional SDR-based method.

Similar results have been provided in Fig. 4, where the transmit power with increased $R$ has been given with required SINR fixed at $18 \mathrm{~dB}$ and $22 \mathrm{~dB}$ respectively. It is worth noting that there exists a trade-off between the power needed for BS and the INR level received by radar. For both SINR levels, the proposed method performs far better than the conventional one especially in all practical INR levels.

The performance of radar has been provided by Fig. 5 and Fig. 6. In Fig. 5, the average detection probability with increased radar SNR is given, where the solid line with diamond markers denotes the case without interference from the BS. Among the rest lines, the solid curves and dashed ones denote the simulated and asymptotic detection performance respectively. The parameters are given as $\eta=13.5 \mathrm{dBm}$, $\Gamma=21 \mathrm{~dB}$, and $P=27 \mathrm{dBm}$. It can be seen that the simulated

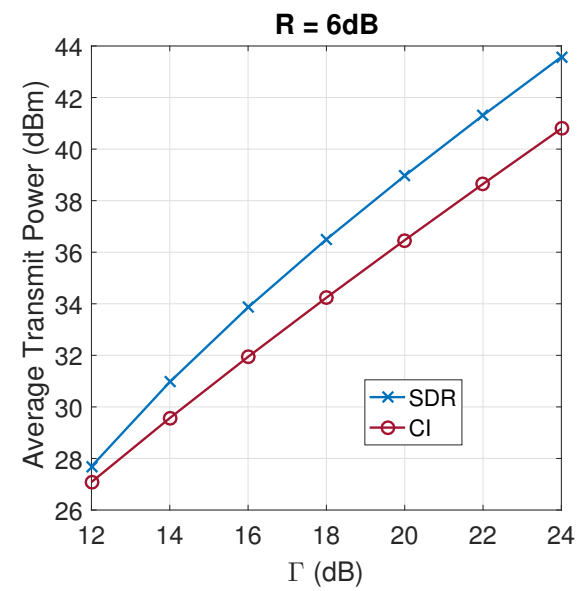

Fig. 3. Average transmit power vs. required SINR, with $R=6 \mathrm{~dB}$.

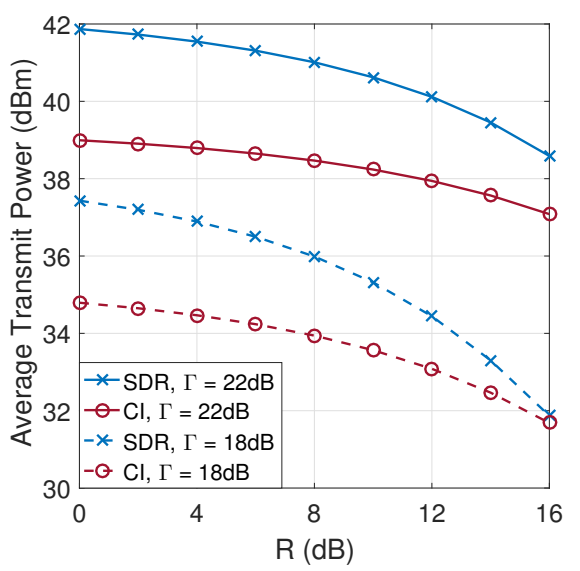

Fig. 4. Trade-off between BS transmit power and INR level, with $\Gamma=18 \mathrm{~dB}$ and $22 \mathrm{~dB}$ respectively.

results match well with the asymptotic ones for both SDR and CI methods. Once again, we see that the proposed method outperforms the SDR-based method significantly. For a desired $P_{D}=0.9$, the extra gain needed for the SDR method is $4.2 \mathrm{~dB}$ compared with the proposed method.

Fig. 6 shows another important trade-off between radar and communication, where the detection probability at the radar with increased $\Gamma$ are provided with $P=24 \mathrm{dBm}, \mathrm{SNR}_{R}=$ $7 \mathrm{~dB}$. The results suggest that a higher SINR requirement at users leads to a lower $P_{D}$ for radar, and the proposed method obtains better trade-off curves for both simulated and asymptotic results thanks to the utilization of MUI. The results in Figs. 5 and 6 justify the use of the Gaussian radar detector of (21) for the CI beamformer, which still gives significant performance gains w.r.t the SDR beamformer.

\section{Conclusion}

This paper proposes a novel optimization-based beamforming approach for MIMO radar and downlink MU-MISO communication coexistence, where multi-user interference 


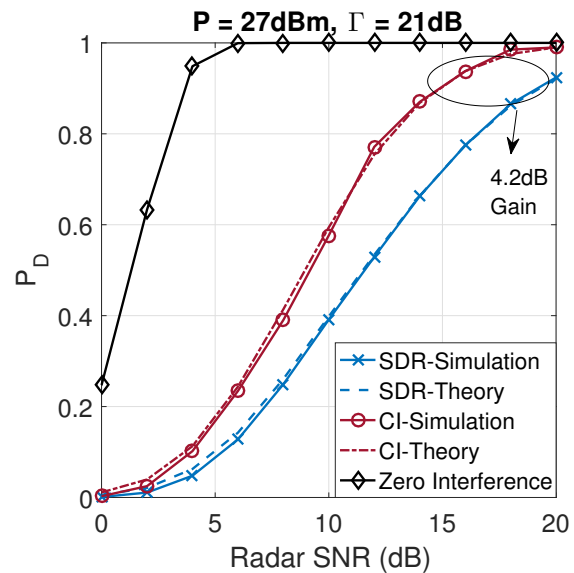

Fig. 5. Detection probability vs. radar SNR for different cases, $P=27 \mathrm{dBm}$, $\Gamma=21 \mathrm{~dB}, \eta=13.5 \mathrm{dBm}$.

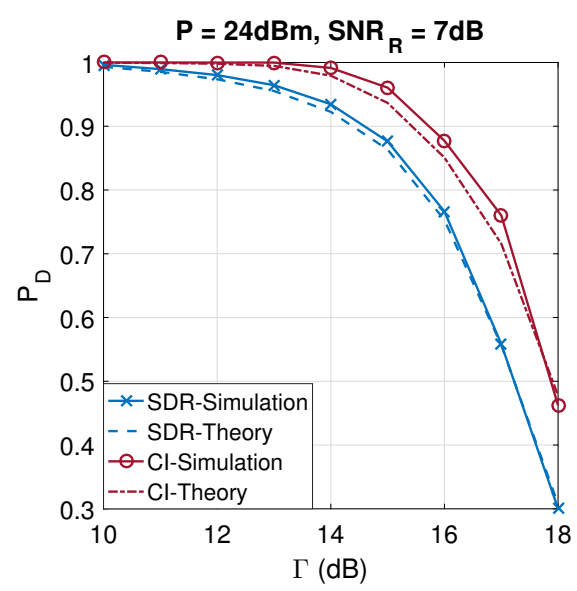

Fig. 6. Trade-off between detection Probability and downlink SINR threshold, $P=24 \mathrm{dBm}, \mathrm{SNR}_{R}=7 \mathrm{~dB}$.

is utilized to enhance the performance of communication system and relax the constraints in the optimization problems. Moreover, the detection probability for MIMO radar in the presence of the interference from BS is analytically derived, and the trade-off between the performance of radar and communication is revealed. Numerical results show that the proposed scheme outperforms the conventional SDRbased beamformers in terms of both power and interference minimization.

\section{ACKNOWLEDGMENT}

This research was supported by the Engineering and Physical Sciences Research Council (EPSRC) project EP/M014150/1 and the China Scholarship Council (CSC) under Grant No. 201606030054.

\section{REFERENCES}

[1] F. C. Commission et al., Connecting America: The national broadband plan, 2010.

[2] J. Chapin, Shared Spectrum Access for Radar and Communications (SSPARC), 2013.
[3] N. S. Foundation, Spectrum Efficiency, Energy Efficiency, and Security (SpecEES): Enabling Spectrum for All, 2016.

[4] R. Saruthirathanaworakun, J. M. Peha, and L. M. Correia, "Opportunistic sharing between rotating radar and cellular," IEEE Journal on Selected Areas in Communications, vol. 30, no. 10, pp. 1900-1910, November 2012

[5] A. R. Chiriyath, B. Paul, G. M. Jacyna, and D. W. Bliss, "Inner bounds on performance of radar and communications co-existence," IEEE Transactions on Signal Processing, vol. 64, no. 2, pp. 464-474, Jan 2016.

[6] S. Sodagari, A. Khawar, T. C. Clancy, and R. McGwier, "A projection based approach for radar and telecommunication systems coexistence," in 2012 IEEE Global Communications Conference (GLOBECOM), Dec 2012, pp. 5010-5014.

[7] A. Khawar, A. Abdelhadi, and C. Clancy, "Target detection performance of spectrum sharing MIMO radars," IEEE Sensors Journal, vol. 15, no. 9, pp. 4928-4940, Sept 2015.

[8] A. Babaei, W. H. Tranter, and T. Bose, "A nullspace-based precoder with subspace expansion for radar/communications coexistence," in 2013 IEEE Global Communications Conference (GLOBECOM), Dec 2013, pp. 3487-3492.

[9] A. Khawar, A. Abdelhadi, and T. C. Clancy, "Coexistence analysis between radar and cellular system in LoS channel," IEEE Antennas and Wireless Propagation Letters, vol. 15, pp. 972-975, 2016.

[10] B. Li and A. Petropulu, "MIMO radar and communication spectrum sharing with clutter mitigation," in 2016 IEEE Radar Conference (RadarConf), May 2016, pp. 1-6.

[11] B. Li, A. P. Petropulu, and W. Trappe, "Optimum co-design for spectrum sharing between matrix completion based MIMO radars and a MIMO communication system," IEEE Transactions on Signal Processing, vol. 64, no. 17, pp. 4562-4575, Sept 2016.

[12] F. Liu, C. Masouros, A. Li, and T. Ratnarajah, "Robust MIMO beamforming for cellular and radar coexistence," arXiv preprint arXiv:1612.03788v3, 2016.

[13] Z. Q. Luo, W. K. Ma, A. M. C. So, Y. Ye, and S. Zhang, "Semidefinite relaxation of quadratic optimization problems," IEEE Signal Processing Magazine, vol. 27, no. 3, pp. 20-34, May 2010.

[14] A. B. Gershman, N. D. Sidiropoulos, S. Shahbazpanahi, M. Bengtsson, and B. Ottersten, "Convex optimization-based beamforming," IEEE Signal Processing Magazine, vol. 27, no. 3, pp. 62-75, May 2010.

[15] C. Masouros, T. Ratnarajah, M. Sellathurai, C. B. Papadias, and A. K. Shukla, "Known interference in the cellular downlink: a performance limiting factor or a source of green signal power?" IEEE Communications Magazine, vol. 51, no. 10, pp. 162-171, October 2013.

[16] C. Masouros and G. Zheng, "Exploiting known interference as green signal power for downlink beamforming optimization," IEEE Transactions on Signal Processing, vol. 63, no. 14, pp. 3628-3640, July 2015.

[17] C. Masouros and E. Alsusa, "Dynamic linear precoding for the exploitation of known interference in MIMO broadcast systems," IEEE Transactions on Wireless Communications, vol. 8, no. 3, pp. 13961404, March 2009.

[18] _ - "Soft linear precoding for the downlink of DS/CDMA communication systems," IEEE Transactions on Vehicular Technology, vol. 59, no. 1, pp. 203-215, Jan 2010.

[19] C. Masouros, "Correlation rotation linear precoding for MIMO broadcast communications," IEEE Transactions on Signal Processing, vol. 59, no. 1, pp. 252-262, Jan 2011.

[20] M. Alodeh, S. Chatzinotas, and B. Ottersten, "Constructive interference through symbol level precoding for multi-level modulation," in 2015 IEEE Global Communications Conference (GLOBECOM), Dec 2015, pp. $1-6$.

[21] A. Li and C. Masouros, "Exploiting constructive mutual coupling in P2P MIMO by analog-digital phase alignment," IEEE Transactions on Wireless Communications, vol. PP, no. 99, pp. 1-1, 2017.

[22] J. Li and P. Stoica, "MIMO radar with colocated antennas," IEEE Signal Processing Magazine, vol. 24, no. 5, pp. 106-114, Sept 2007.

[23] I. Bekkerman and J. Tabrikian, "Target detection and localization using MIMO radars and sonars," IEEE Transactions on Signal Processing, vol. 54, no. 10, pp. 3873-3883, Oct 2006.

[24] S. Boyd and L. Vandenberghe, Convex optimization. Cambridge university press, 2004.

[25] S. M. Kay, Fundamentals of statistical signal processing: Detection theory, vol. 2. Prentice Hall, 1998. 